\title{
Genome-wide survey and expression analysis of the OSCA gene family in rice

Yunshuang Li ${ }^{1}$, Fang Yuan², Zhaohong Wen ${ }^{1}$, Yihao Li ${ }^{1}$, Fang Wang ${ }^{1}$, Tao Zhu', Wenqing Zhuo ${ }^{1}$, Xi Jin ${ }^{1}$, Yingdian Wang ${ }^{1}$, Heping Zhao ${ }^{1}$, Zhen-Ming Pei ${ }^{2}$ and Shengcheng Han $^{1 *}$

\begin{abstract}
Background: Reception of and response to exogenous and endogenous osmotic changes is important to sustain plant growth and development, as well as reproductive formation. Hyperosmolality-gated calcium-permeable channels (OSCA) were first characterised as an osmosensor in Arabidopsis and are involved in the perception of extracellular changes to trigger hyperosmolality-induced $\left[\mathrm{Ca}^{2+}\right]_{\mathrm{i}}$ increases $(\mathrm{OICl})$. To explore the potential biological functions of OSCAs in rice, we performed a bioinformatics and expression analysis of the OsOSCA gene family.

Results: A total of 11 OsOSCA genes were identified from the genome database of Oryza sativa L. Japonica. Based on their sequence composition and phylogenetic relationship, the OsOSCA family was classified into four clades. Gene and protein structure analysis indicated that the 11 OsOSCAs shared similar structures with their homologs in Oryza sativa L. ssp. Indica, Oryza glaberrima, and Oryza brachyantha. Multiple sequence alignment analysis revealed a conserved DUF221 domain in these members, in which the first three TMs were conserved, while the others were not. The expression profiles of OsOSCA genes were analysed at different stages of vegetative growth, reproductive development, and under osmotic-associated abiotic stresses. We found that four and six OsOSCA genes showed a clear correlation between the expression profile and osmotic changes during caryopsis development and seed imbibition, respectively. Orchestrated transcription of three OsOSCAs was strongly associated with the circadian clock. Moreover, osmotic-related abiotic stress differentially induced the expression of 10 genes.

Conclusion: The entire OSCA family is characterised by the presence of a conserved DUF221 domain, which functions as an osmotic-sensing calcium channel. The phylogenetic tree of OSCA genes showed that two subspecies of cultivated rice, Oryza sativa L. ssp. Japonica and Oryza sativa L. ssp. Indica, are more closely related than wild rice Oryza glaberrima, while Oryza brachyantha was less closely related. OsOSCA expression is organ- and tissue-specific and regulated by different osmotic-related abiotic stresses in rice. These findings will facilitate further research in this gene family and provide potential target genes for generation of genetically modified osmotic-stress-resistant plants.
\end{abstract}

Keywords: OSCA, DUF221 domain, Phylogenetic relationships, Expression profile, Osmotic stress, Oryza

\section{Background}

Drought and salt stress are major abiotic constraints affecting plant growth worldwide. The first phase common to drought and salt stress is osmotic stress [1]. Because of their sessile lifestyle, plants have developed mechanisms to avoid or cope with the consequences of water stress. Previous studies showed that plants have developed different signal transduction pathways and

\footnotetext{
* Correspondence: schan@bnu.edu.cn

${ }^{1}$ Beijing Key Laboratory of Gene Resource and Molecular Development, College of Life Sciences, Beijing Normal University, Beijing 100875, China Full list of author information is available at the end of the article
}

gene expression regulation mechanisms to perceive and respond to water deficiency [2-4]. The mechanism of the response to drought included both abscisic acid (ABA)-independent and ABA-dependent signalling cascade pathways, as well as the expression of droughtrelated genes, such as DREB and NAC [5-7]. The ABAresponsive element (ABRE) and its binding transcription factors are involved in ABA-dependent gene expression. Similarly, the dehydration-responsive element (DRE) and its binding protein 2 transcription factors play pivotal roles in ABA-independent gene expression in response to osmotic stress [7]. ABA synthesised after water deficit 
potently inhibits stomatal opening and promotes stomatal closure to prevent water loss. In addition, ABA-activated gene expression is associated with plant adaption to drought, involving genes such as RD22, RD29A, KIN1, and KIN2 [8]. However, the mechanism underlying the early response to osmotic stress in plants remains undiscovered.

The early events of plant adaptation to drought stress include perception of osmotic changes and consequent stress signal transduction cascades, leading to the activation of various physiological and metabolic responses, including stress responsive gene expression. A total of 16 cDNAs of the early response to dehydration (ERD) genes were isolated from Arabidopsis after treatment with dehydration for $1 \mathrm{~h}$ [9]. ERD4 encodes a protein containing a highly conserved DUF221 domain (domain of unknown function 221), which is common to various species [10-12]. On the other hand, osmotic stress and various other stimuli trigger increases in the cytosolic/ intracellular free calcium concentration $\left(\left[\mathrm{Ca}^{2+}\right]_{\mathrm{i}}\right)$ in plants $[13,14]$. The hyperosmolality-induced $\left[\mathrm{Ca}^{2+}\right]_{\mathrm{i}}$ increase $(\mathrm{OICI})$ occurs within $5 \mathrm{~s}$, which may be the earliest detectable event in plants [13]. Blocking OICI disrupts drought and ABA-induced gene expression, suggesting that the precise regulation of OICI is crucial for activation of many signal transduction pathways triggered by external stimulation; this process is important in understanding plant sensing of external osmotic stress and other stimulations. Previous studies showed that osmotic/mechanical stimuli-gated $\mathrm{Ca}^{2+}$-permeable channels serve as osmosensors in bacteria and animals $[15,16]$, which indicated that OICI in plants is mediated by specific calcium permeable channels that function as osmosensors.

Using a calcium-imaging-based unbiased forward genetic screening strategy, Yuan et al. isolated several Arabidopsis mutants (osca1) that showed low OICI, and further characterised OSCA1 as a previously unknown hyperosmolality-gated calcium-permeable channel, suggesting that OSCA1 may be an osmosensor in Arabidopsis [17]. OSCA1 belongs to a gene family with 15 members in Arabidopsis, and homologues are found in other plant species and throughout eukaryotes. In this family, OSCA3.1 encoded an ERD4 protein [9]. Yuan et al. also reported that OSCA3.1-knockout mutants displayed normal OICIs, suggesting that OSCA3.1 may differ from OSCA1, reminiscent of the diverse functions of TRPs (transient receptor potential channels) in animals [17].

In the present study, we characterised OSCA family members in four species of the Oryza genus in silico and analysed the phylogenetic relationships among these OSCAs, as well as their expression profiles in various organs/tissues and under different osmotic-related abiotic stresses. These results can be used for functional validation studies of the rice OSCA genes and increase our understanding of the roles of plant OSCAs.

\section{Results}

Identification of $O S C A$ genes

To explore the entire OSCA gene family in rice, we used the sequence of 15 AtOSCAs to search against the Oryza sativa L. ssp. Japonica genome in RGAP (Rice Genome Annotation Project) and the genome of Oryza sativa L. ssp. Indica, Oryza glaberrima, and Oryza brachyantha from the Ensembl Genomes database at the E-value of 1e-10. The presence of conserved DUF221 domain in their protein structure is the excusive criterion to confirm the OSCAs with The SMART program (The Simple Modular Architecture Research Tool). By removing sequence redundancies and alternative splice forms of the same gene, we identified 11 putative $O S C A$ genes and named them OsOSCA1.1 to OsOSCA4.1, in accordance with Arabidopsis orthologues (Additional file 1: Table S1). OsOSCA3.1 was OsERD4, as reported previously [10]. Next, we identified 11 OsIOSCAs, 12 OgOSCAs, and 11 ObOSCAs in Oryza sativa L. ssp. Indica, Oryza glaberrima, and Oryza brachyantha, respectively (Additional file 1: Table S1). There are two orthologues of OSCA4.1 in Oryza glaberrima, named OgOSCA4.1_1 and OgOSCA4.1_2, which was indicative of gene duplication after Oryza glaberrima had split from the rice species.

\section{Phylogenetic relationship and gene structure of rice OSCAs}

To compare the evolutionary relationship of OSCAs among four rice species, a phylogenetic tree was generated using the CDS (Sequence coding for amino acids in protein) of 45 OSCAs. We found that members of the OSCA family were separated into four distinct clades, designated I, II, III, and IV (Fig. 1a). Clade I included four members-OSCA1.1, OSCA1.2, OSCA1.3, and OSCA1.4; Clade II contained five members-OSCA2.1, OSCA2.2, OSCA2.3, OSCA2.4, and OSCA2.5; Clades III and IV contained only OSCA3.1 and OSCA4.1, respectively. The OSCA gene phylogenetic tree revealed that two subspecies of cultivated rice, Oryza sativa L. ssp. Japonica and Oryza sativa L. ssp. Indica, were more closely related than wild rice Oryza glaberrima, while Oryza brachyantha was less closely related. Excluding clade IV, which contained five orthologue members, all other clades contained four orthologue, suggesting that the OSCA gene had duplicated, resulting in different members of the family in the ancestral species before speciation.

Previous studies have shown that the exon/intron diversification among gene family members plays an important role in the evolution of multiple gene families 


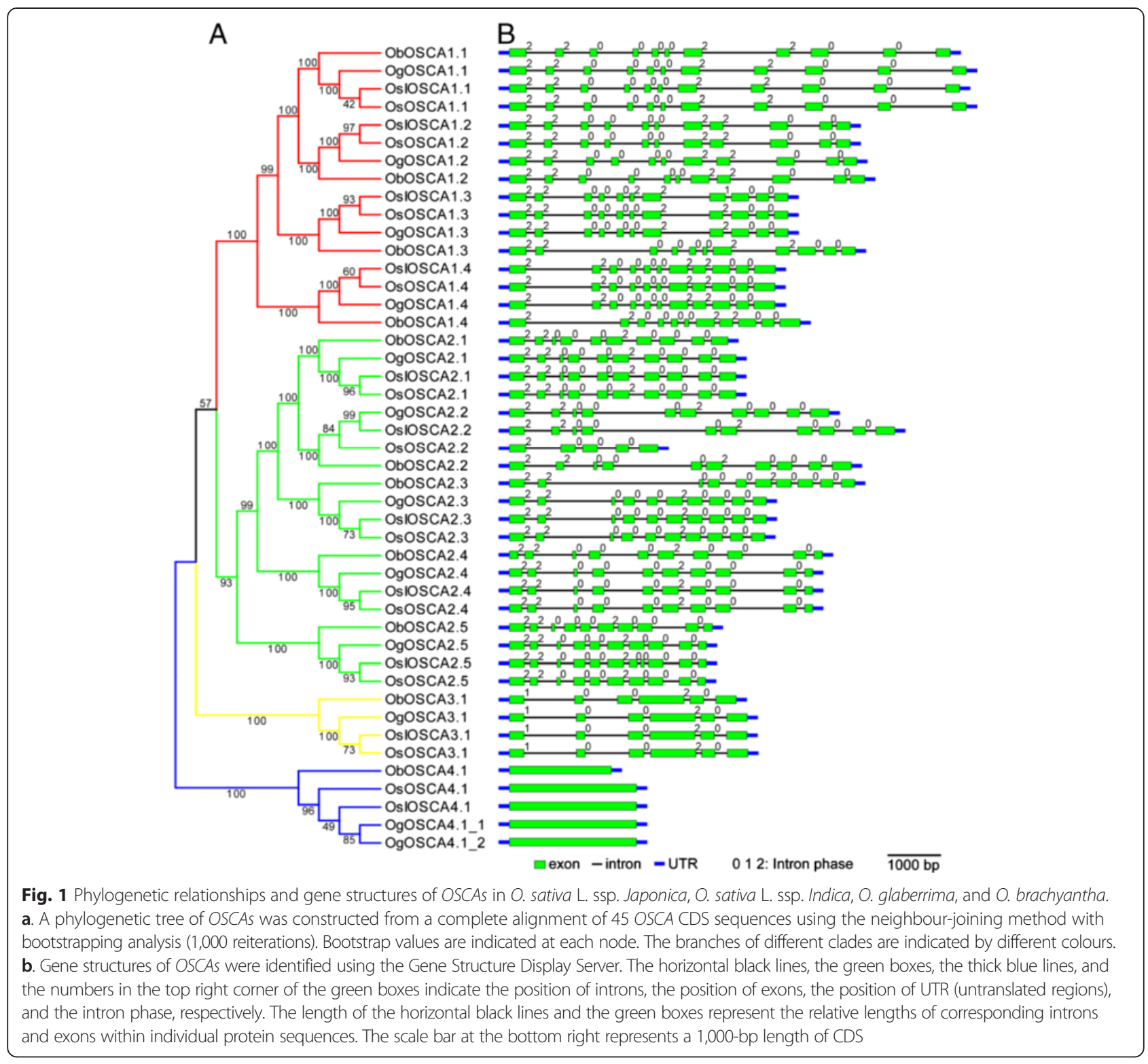

through three main mechanisms: exon/intron gain/loss, exonisation/pseudoexonisation, and insertion/deletion [18]. The numbers and positions of exons and introns in OSCAs were determined by comparing full-length cDNA sequences and the corresponding genomic DNA sequences of each OsOSCA gene using Gene Structure Display Server 2.0 (http://gsds.cbi.pku.edu.cn/). We found that all OSCA genes contained multiple exons with the exception of OSCA4.1. Moreover, in the same clade in the phylogenetic tree, most members shared almost identical intron/exon structures and intron phases (Fig. 1b). This finding further validated the nomenclature proposed by our phylogenetic analysis, and the main structural characteristics in the gene and protein sequence of OSCAs were formed prior to the split between wild and cultivated rice. However, further studies are required to elucidate the specifics of a functional divergence among the OSCA genes.

\section{The conserved domain of OSCAs}

We used the SMART to confirm the structural characterisation of the OSCAs and found that most OsOSCAs contained four main modular architectures, the transmembrane helices (TM) region, the low-complexity region, a coiled-coil region, and the DUF221 domain, although the number of amino acids in the Oryza genus varied from 481 to 812 (Additional file 5: Figure S1). The TM in OSCAs were predicted using TMHMM Server v. 2.0. We found that different OSCA members contained 6-10 TMs, and the same OSCA orthologue in 
the four rice species contained the same number of TMs with three exceptions. OsOSCA2.2 and ObOSCA4.1 had three TMs fewer than their orthologues, which suggested that a deletion event occurred in the genomes of OsOSCA2.2 and ObOSCA4.1; OSCA2.5 had also two TMs fewer in two subspecies of Oryza sativa L. than its orthologues in Oryza glaberrima and Oryza brachyantha. However, there is no deletion in the genomes of OsOSCA2.5 and OsIOSCA2.5 (Additional file 1: Table S1 and Additional file 5: Figure S1).

The entire OSCA family is characterised by the presence of a conserved DUF221 domain, which functions as an osmotic-sensing calcium channel [17]. According to InterPro (http://www.ebi.ac.uk/interpro/) Pfam, DUF221 represents the seven transmembrane domain region of calcium-dependent channel and is homologous to domains in anoctamin/TMEM16 channels, which are calcium-activated chloride channel $(\mathrm{CaCC})$ components [19], and salt taste chemosensation transmembrane channel-like (TMC) proteins in C. elegans [20] or mechanosensitive TMCs in hair cells of the mammalian inner ear [21]. Multiple sequence alignment was performed to clarify the characteristics of DUF221 in 11 OsOSCAs (Fig. 2). In general, the core region of DUF221 contained four to six TM regions; TM1-TM3 were highly conserved in all OsOSCAs, while TM4-TM6 were not. We also identified 11 conserved amino acid residues in the DUF221 region of OsOSCAs, A319, V321, F323, A329, A349, P350, W357, L425, P426, F467, and Y613 of OsOSCA1.1, which could be associated with the channel characteristics of OSCAs.

\section{Expression analysis of OsOSCA in various organs}

To unveil the potential function of OsOSCAs in rice, the expression profiles of OsOSCA genes in various tissues and organs were first determined using qRT-PCR (realtime reverse transcription-PCR) and represented in grey scale to facilitate visualisation: 30-day-old root (Rt); 30day-old shoot (St); mature stem (Sm); mature flag leaf $(\mathrm{Fl})$; stamen $(\mathrm{Sn})$; pistil $(\mathrm{Pi})$; and mature seed $(\mathrm{Sd})$. The 11 OsOSCA genes showed tissue-specific expression patterns (Fig. 3). Five genes, OsOSCA1.1, OsOSCA1.2, OsOSCA2.4, OsOSCA3.1, and OsOSCA4.1, were highly expressed in all tissues tested, which was indicative of a universal role of these OsOSCAs in osmotic-sensing processes throughout the plant. OsOSCA2.2 and OsOSCA2.5 showed medium transcript abundances in all tissues tested. OsOSCA2.3 was detected only in the stamen, indicative of a specific function therein. OsOSCA1.3 and OsOSCA1.4 had relatively higher transcript abundance in the stamen and low transcript abundance in other tissues. OsOSCA2.1 had high transcript abundance in the shoot and stamen, but low levels in other tissues. These results indicated that the OsOSCA genes were involved in various physiological and developmental processes in rice.

\section{Expression of OsOSCAs during caryopsis development}

To explore the transcriptional expression of OsOSCAs during caryopsis development after pollination, we extracted expression data of OsOSCAs from a published microarray database (http://signal.salk.edu/cgi-bin/RiceGE, GSE6893) and re-analysed their expression levels during various rice reproductive developmental stages, including panicles at different stages (P1-P6) and developmental seeds after pollination (S1-S5). At least one probe for each OsOSCA was present on the rice whole genome Affymetrix array platform (GPL2025). The 11 OsOSCA genes were divided into three subgroups, i-iii, according to their similar expression patterns (Additional file 3: Table S3 and Fig. 4a). OsOSCA1.1, OsOSCA2.4, and OsOSCA3.1 in subgroup i were expressed with high abundance in all reproductive developmental stages of rice. Subgroup ii contained three genes, OsOSCA1.3, OsOSCA2.3, and OsOSCA2.5, expressed with very low abundance in almost all tissues examined. Subgroup iii included the remaining five OsOSCAs and showed medium abundance in all organs. More interestingly, several OsOSCAs, including OsOSCA1.4, OsOSCA2.4, OsOSCA2.5, and OsOSCA4.1, showed gradually increased expression levels during seed development, which was confirmed by qRT-PCR analysis of the expression levels of OsOSCA genes during caryopsis development (Fig. 4b). However, we found that the transcript levels of OsOSCA1.1, OsOSCA1.2, and OsOSCA1.3 were increased in the developing caryopsis from the middle stage of caryopsis development (8 days after pollination) to the last stage (30 days after pollination). A decrease in OsOSCA2.2 and OsOSCA3.1 transcript levels was detected in the caryopsis from the earliest to the last stage of caryopsis development. OsOSCA2.3 transcript levels were higher in the caryopsis during the earliest and middle stages of development, while the expression of OsOSCA2.1 was unchanged during caryopsis development (Fig. 4b). We used the relative water content, which showed gradually decreased, as a control for caryopsis development (Additional file 6: Figure S2).

\section{Expression of OsOSCAs in the progress of rice seed imbibition}

Imbibition is the first and essential phase for seed germination. Water content gradually increases in seeds during this period, leading to a less negative water potential. Thus, it was important to explore whether the expression of OsOSCAs corresponded to osmosis variation during seed imbibition. We found that the expression levels of OsOSCA1.1, OsOSCA1.2, OsOSCA2.1, OsOSCA2.4, OsOSCA2.5, and OsOSCA4.1 
were decreased during seed imbibition, from the start until $20 \mathrm{~h}$, while the water content increased (Fig. 5 and Additional file 7: Figure S3). Furthermore, the lower OsOSCA transcription levels were in accordance with the increased water content during seed imbibition in $18 \%$ and $30 \%$ PEG (polyethylene glycol) 6000 solutions, respectively. Thus, the transcription of most OsOSCA genes was correlated with the water potential in imbibed seeds, which indicated that OsOSCAs play an important role in sensing and/or responding to osmotic changes to regulate seed germination.

\section{Orchestrated transcription of several OsOSCAs by the circadian clock}

Water in plants is transported primarily from the root to the shoot through the transpiration stream driven by evaporation. The transpiration rate is governed by stomatal conductance, which displays diurnal oscillations $[22,23]$. Thus, the water potential in the stomatal apoplast is synchronised to stomatal conductance oscillations, which may determine the circadian expression of OsOSCAs. To test this hypothesis, we analysed the expression profiles of all OsOSCAs in the shoots of fourleaf-stage rice seedlings under 14 -h light $\left(24^{\circ} \mathrm{C}\right) / 10-\mathrm{h}$ dark $\left(20{ }^{\circ} \mathrm{C}\right)$ conditions, and found that OsOSCA1.2, OsOSCA2.1, and OsOSCA2.2 were subjected to a circadian rhythm at the transcriptional level (Fig. 6). During the day, stomata opening results in water loss via transpiration and higher water potential in the apoplast, which may gradually decrease the expression of OsOSCA1.2, OsOSCA2.1, and OsOSCA2.2. Conversely, stomata closing at night will trigger the transcription of these three OsOSCAs, which will peak following the dark to light transition. Except those three OsOS$C A s$, the expression of others was independent of the circadian rhythm. We used OsLHY, which exhibited robust rhythmic expression under diurnal conditions, as a positive control in this experiment (Additional file 8: Figure S4) [24].

\section{Expression profiles of OsOSCAs under osmotic-related abiotic stresses}

To determine whether the expression of OsOSCAs is responsive to osmotic-related abiotic stress, qRT-PCR analysis of the OsOSCAs at the four-leaf stage in rice was performed under different stress treatments: PEG 6000 (20\%), $\mathrm{NaCl}(150 \mathrm{mM})$, drought, and ABA (100 mM). We found that nine OsOSCA genes were down- or upregulated $(<0.5$ or $>2)$ in at least one of the stress conditions examined as compared with the control, except for OsOSCA2.2 and OsOSCA2.3 (Fig. 7). In detail, the expression of five genes, OsOSCA1.2, OsOSCA2.1, OsOSCA2.4, OsOSCA2.5, and OsOSCA3.1, were upregulated by all four kinds of treatment.
OsOSCA4.1 was upregulated by PEG and salt stress as well as ABA treatment, while OsOSCA1.1 was upregulated by PEG and salt stress. We also found that OsOSCA1.4 was specifically downregulated by drought stress and upregulated by ABA treatment. OsOSCA1.3 was downregulated after PEG stress and ABA treatment. The expression of marker genes, PER24P for PEG treatment [25], DSM2 for salt [26], OsP5C for drought [27], $A B I 5$ for ABA [28], is shown in Additional file 9: Figure S5. We also investigated the expression of three housekeeping gene: actin (LOC_Os03g61970.1), eEF1a (LOC_Os03g08020) and UBQ5 (LOC_Os01g22490) under different abiotic stresses and calculated the Gene expression stability values (M) of these three genes, which was 0.753 for actin, 0.841 for eEF1a and 1.069 for $U B Q 5$, respectively (Additional file 4: Table S4). The $M$ value of three genes is below the threshold value of 1.5, which showed that actin gene is suitable for using as the internal controls to normalize the expression of OSCA genes in rice. These results indicated that OsOSCAs might be involved in osmotic-related signalling pathways and play pivotal roles in the responses to various abiotic stresses in rice.

\section{Discussion}

During their life cycle, plants encounter a variety of exogenous and endogenous osmotic changes and have developed various strategies to sense, respond, and adapt to these stresses. Exogenous osmotic stress includes drought, salt, temperature and the water potential in the stomatal apoplast, which is regulated by stomatal conductance. Endogenous osmotic stimuli are caused by material accumulation or consumption, such as caryopsis development, seed maturation, and seed imbibition during germination. In recent studies, OSCA was identified as an osmosensor mediating hyperosmolarityinduced cytosolic calcium increases (OICI) in Arabidopsis, which increased our understanding of the molecular mechanisms underlying sensing of osmotic stresses by plants [17, 29].

Oryza (23 species; 10 genome types) contains the world's most important food crop, rice, which has diversified across a broad ecological range, from deep water to upland, including seasonally dry habitats. This diversification occurred within a narrow evolutionary time scale ( 15 million years) due to several closely spaced speciation events, constituting an almost stepwise historical genomic record [30, 31]. Therefore, studying the phylogenetic relationship of OSCAs in four Oryza species and the expression levels of OsOSCA family genes in various tissue/organs, developmental stages, and under various abiotic stresses will facilitate further research on this gene family and provide 




OSOSCA1.1 YLTIRRLI IAVAFFFLNFFYVLPIAFVQSLA. . . . . NIEGIEKAAPFLKPLIEMRT IKS FIOG HLP. GIALKIF

OSOSCA1.2 SLTVRRLIVAVAFFELNFFYVIPIAFVQSLA. . . . . SLEGIEKALPFLKPL IKIDVIKS FIOGELP. GIALKVE

OsOSCA1.3 ELSVRRL IMAVALFFLT FF FMIP IAIVQSMA . . . . . NLDD IERMLP FLKP I IERNS LKS IVOGFLP. GIALKI

OsOSCA1.4 SLSIRKFLIS IAVFALVFFYMIP IAFVQSLA . . . . NLEG IEKVAP FLRPVIDTPVVKS FLOGELP. GLALKI

OSOSCA2.1 QLWIRR IATLLGS IVFMLFFLIPVTF IQGLS . . . . QLEQLQRLPFLKG ILEKKYMSQLVTGYLP. SVILQIF

OSOSCA2.2 QLWIRR IVTLSGS IVFMFLFL I PVTF IQGLT $\ldots \ldots$ QLEQLQRLP FLNG I LKKKYITQLVTGYLP.SVILQI

OsOSCA2.3 QIWLRKIATLAASVAFMFVFIVPVAFVOSMM $\ldots \ldots$ QLDQIEQLFPSLKNMLKKP FFVKLVT GYLP. SVVLLLS

OsOSCA2.4 DRWISKFVVSVAS ILIILVFLLVSAFVQGLT . . . . YMEQLETWLP FLRNI LE IAVVSQLVTGYLP. SVILHFI

OsOSCA2.5 RIAIYKLGVFIAAFLLIVFFTIPVTAVQGIV $\ldots \ldots$. FEKIKIWFPPARAVELI PGLNSVVTGYLP. SMILNGF

OSOSCA3.1 ERQIRQVVVYTIVELTVVFYMIPITAISALT . . . . TLEKLREKLPFLKVVVDQPKIKTVLQAYLP. QLALIVF

OSOSCA4.1 SLGLRR IAVNTCLILMLLFESSPLA I ISGMQSAAR I INVEAMDNAKSWLVWLQSSSWEWT I IFO LPNVLI FVSM

Consensus

OsOSCA1.1 LLLIPSTLMEMSKVEGLTSVSSLERRSAFKYYIFLFFNVELGS IIAGSALEQLKTFLHQSANE. . . . . IPRTIG 502 OSOSCA1.2 LILIPTILMFMSKFEGLISOSSLERRSASKYYIFLFENVELGSIVTGSALDQLKAYIHQSANE. . . . IPRTIG 502

OsOSCA1.3 LILIPTFLVMMSKIEGHTSLSGLDRRTASKYYIFLFVNVELGSVITGTAFQLLNNFIHQSANK...... IPEIVG 503

OsOSCA1.4 LYILPTVLMIMSKVEGYVSLSSLERRAASKYYYFMLVINVELGS I IAGTAFEQLNAFFHQPPSQ . . . . IPRTIG 500

OsOSCA2.1 LYAVAPIMILESTLEGPISHSERKRSACCKVLYFTVWNIFEGNVLSGTVISQL. NVLSSPKD . . . . . IPVQLA 485

OSOSCA2.2 LXTVPPTMMFFSTLEGPVSHSERKRSACCKVLYFT IWNVEFVNVLSGSAISQV.NALSSPKD . . . . IPMVLA 188

OSOSCA2.3 LYTVPPIMMFESSIEGS ISRSGRKKSACCK ILFETIWNVFFVNVLSGSVLNQL. NVETRPRD . . . . . MPSMLA 485

OsOSCA2.4 SSYVPSTMKLESTMQGFISVSGIERSACNKMLRFTIWSVFFANVLTGSVLGQL.EIFLDPKE. . . . IPKRLA 471

OsOSCA2.5 IYLIPEAMLGMASEEGCIAKSOKE IKACNMVFY FLLGNVFFLS ILSGSLLHQIGESFTHPKD . . . . . IPSR LA 482



OsOSCA4.1 YIIIPSVLSYFSKFECHLTVSGEQRAALIKMVCFELVNLILLRALVESSLESWILSMGRCYLDSVDCKQ IEQYLS 533

Consensus

OsOSCA1.1 EAIPMKATFF. ITYVMVDGWAGVAGEILRLKPLI I FHLKNFFLVKTËKDREEAMDPGS I GËDSNEPQPIQLY FLLG 576

OSOSCA1.2 VAIPMRATFF. I TYVMVDGWTGVAGEILRLRALI I FHLKNFFLVKTEKDREEAMDPGS ICFDWCEPRIOLYFLLG 576

OsOSCA1.3 ESIPMKATEF. ITYVMVDGWAGVAAEVLRLKPLVMFHIKNT FLVRTSR. REQAMDPGSLDFGTTEPRIQLYELLG 576

OsOSCA1.4 VAIPMKATFF. MTY IMVDGWAGIANEILRVKPLVIYHLKNMFIVKTERDRERAMD PGS I GLAENLPSLQLY FLLG 574

OSOSCA2.1 RAIPVQATFF. ITYVLTSGWASLSSELMQLFGLIWNFVRKYILRMPEDTEFVPSFP. . . Y YHTEVPKVLLFGLLG 555

OSOSCA2.2 RAVPVQATFF. TTYVLTSGWASLSSELMOLFGLTWNFIMKYVLRMKEDSYFVPSFP. . . Y YHTEVPKVLLFGLLG 258

OSOSCA2.3 ELVPKQATEF. ITYVLTSGWASLCSEILOVYNLVYNFFRKCIFCYRDDPEYGYSFP. . . . YHTEVPKVLIFNLLG 555

OSOSCA2.4 VVVPAQASEF. ITYVVTS.WTSIASELTQTAALLFHLWGSCAKCCKRDESKPPSMH.... YHSEIPRVLLEGLLG 540

OsOSCA2.5 RAVSAQSDEF.ITYILTDGMSGFSLEVLQFGLLTWHFFKAHSIGHSEQP. YLYGFP. . . YYRVVPIVSLAVLIG 551

OsOSCA3.1 SSI PGSATFF, LTFVALKFFVGYGL ELSRLVPLI I FHLKRKYLCKTEDEVRAAWA PGDLGYNTRVPNDMLIVTTV 577

OSOSCA4.1 PSFLSRSSLSSLAELITCTFLGISFDLLAPI PWIKHVMKKFRKNDMVQLVPEENEDYQLMHDGEETNNLRAPLMS 608

Consensus

OsOSCA1.1

TM5

TM6

FILIFEG GAYVVYRHQ I INVYNQEYES SAAAFWPSVHGR I IVALIVSOLLLLGL ... ISTKKGA 647

OSOSCA1.2. . LVYAVVTPLLLPEILVFFGLAYVVYRHQI INVYNQ QYESGAQFWPSVHGR I I IALIVSOLLLIGL. . . LSTKGF 647

OSOSCA1.3 .LVYAVVTPILLPFIIVEFSLAYLVFRHQI INVYNQQYEESGAQFWPDVQRRLVIALIVSQILLIGL. . . LSTQEA 647

OSOSCA1.4 . LVYAVVTP ILLPFIIIFEAFAFLVYRHQI INVYNQEYESAAAFWPQVHSRIIASLLISHVTLFGL . . MSTMKA 645

OsOSCA2.1 .FTCSVLAPLILPFLLVYFELGYIVYRNQLLNVYRTRYDTGGLYWP IAHNAVIFSLVLTQIICLGV . . FGLKES 626

OsOSCA2.2 .FTCSVLAPLILPFLLVYFELGYVVYRNQFLNVYCTKY T GGLYWP IAHYTT IFS IVLTEI ICLGV . . FGIKES 329

OsOSCA2.3 .FTES IMAPL I LPFLLVYFCLGYLVYRNQ I LNVYY PKYEMGGKLWP IMHSTLVEALVLTOTIALGV . . FT IKHA 626

OsOSCA2.4 .LTYEIVSPLILPEVLVYFCLGYFIYRNQLFNVYS PKY T GGR WWPIVHGGTIFSLVLMHVIAIGV . . F FIKKL 611

OSOSCA2.5 . LVYAVVAPLLLPILVIYFLLGYAVYINQMEDVYE I TYDTCGQYWPNIHRY IFLSVTLMQITML . . . . . . KSK 617

OsOSCA3.1 . LCYSVIAPLI I PEGVAYFALGWI IVKNQVLRVYVP SYESNGRMWPHMHTR I IAALLIYQITMVGV . . ILIKKF 648

OsOSCA4.1 EREDSGILNGIEEHDLSLYPINRS FHMPKQT FDEAQYYAFD I IFALTMIYSLFAPLTVPVGAVYEGYRYLVDKY 683

Fig. 2 (See legend on next page.) 
(See figure on previous page.)

Fig. 2 Multiple sequence alignment and transmembrane region of the DUF221 conserved region in OsOSCAs. Multiple sequence alignments of OSCAs were performed using DNAman and the transmembrane region of the DUF221 conserved region was predicted using TMHMM. The region between two vertical red lines represents the DUF221 conserved region. Identical (100 \%), conserved (75-99 \%), and blocks (50-74 \%) of similar amino acid residues are shaded in dark navy, pink, and cyan, respectively. Identical or similar amino acid residues are shown in lowercase abbreviations at the bottom of the corresponding rows. The transmembrane regions are marked by black lines and called TM1-TM6

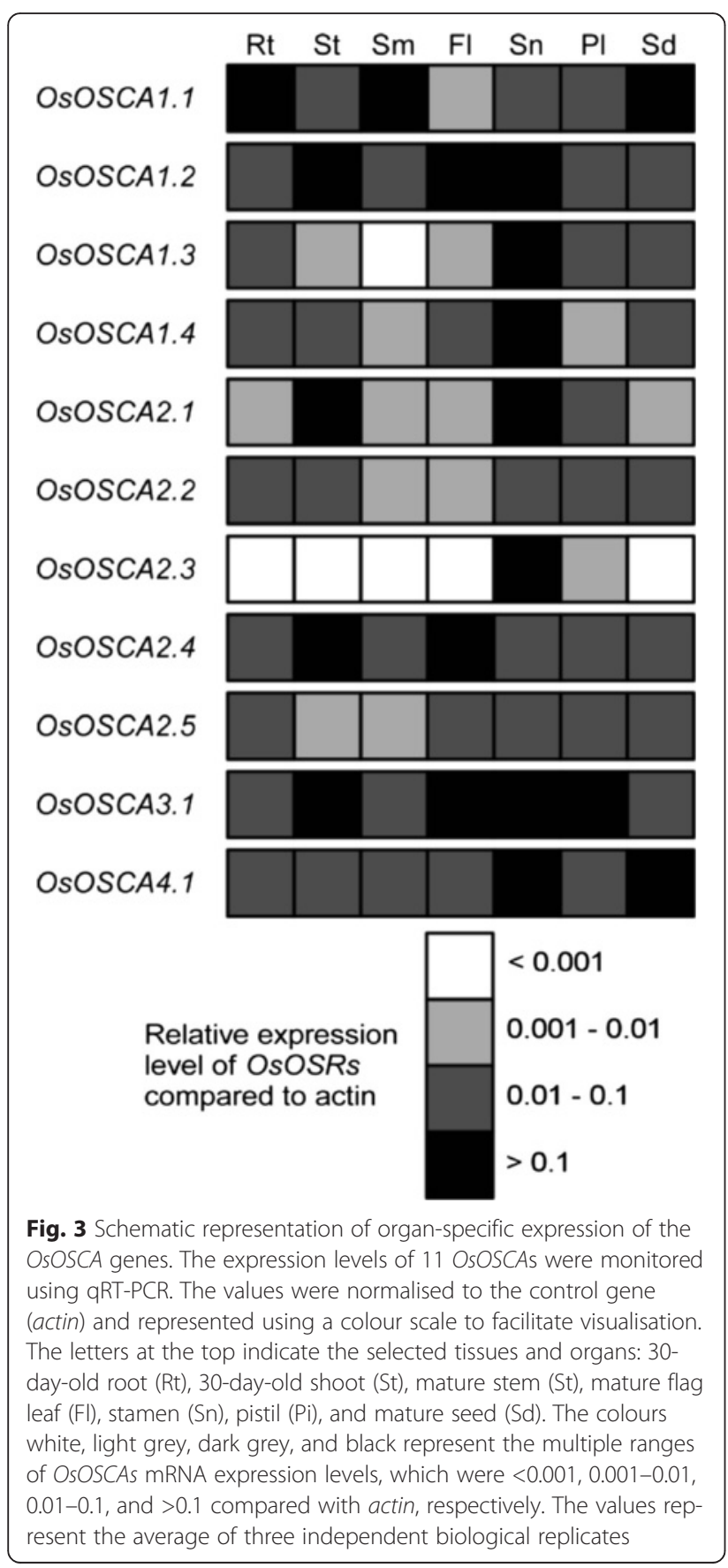

potential target genes for generation of genetically modified osmotic-stress-resistant plants.

Based on the phylogenetic tree, we found that the OSCA genes from two Oryza sativa subspecies, with the exception of OSCA2.2, were clustered more closely with their orthologues from Oryza glaberrima than those from Oryza brachyantha, which indicated that Oryza brachyantha and Oryza glaberrima split long before the separation of cultivated and wild rice. Furthermore, the subspecies of cultivated rice, Oryza sativa L. ssp. Japonica and Oryza sativa L. ssp. Indica, have the closest relationship, which further supported the evolutionary origins in diploid Oryza. With the same AA genome species as Oryza sativa, the wild rice Oryza glaberrima is more closely than Oryza brachyantha because it is an FF genome species [30]. OsOSCA2.2 in Oryza sativa L. ssp. Japonica lacks the first five exons compared with its orthologues in Oryza sativa L. ssp. Indica, Oryza brachyantha, and Oryza glaberrima, which accounts for the predicted protein structure of OsOSCA2.2 lacking the first three TM regions. In addition, OSCA4.1 contains a single exon and ObOSCA4.1 is shorter than its three homologues. This leads to the absence of the first three TM regions in ObOSCA4.1, which are present in the homologous proteins in the other three rice species. These results suggested that OSCA4.1 is the most conserved member of the OSCA family, and that deletions in OsOSCA2.2 and ObOSCA4.1 occurred independently during rice evolution. Furthermore, we predicted that the first three TM regions may not be essential for the basic ion channel activity of OSCAs, but essential for osmosensor specificity.

In this study, we found that OsOSCA genes were expressed in tissue-specific patterns, indicative of a specific role for each member of the OsOSCA family in sensing various osmotic-related stresses by different tissues/organs. In addition, it was well known that osmotic conditions appear to control seed development in many plant species [32]. During caryopsis development and seed maturation after fertilisation, material accumulation and decreasing water content result in an increasing osmotic potential in endosperm cells, which may regulate the transcriptional expression of OSCAs. This study demonstrated that the transcription of OsOSCA1.2, OsOSCA1.3, and OsOSCA2.5 was in accordance with increased endogenous osmotic changes during rice caryopsis development. In contrast, osmotic potential was 


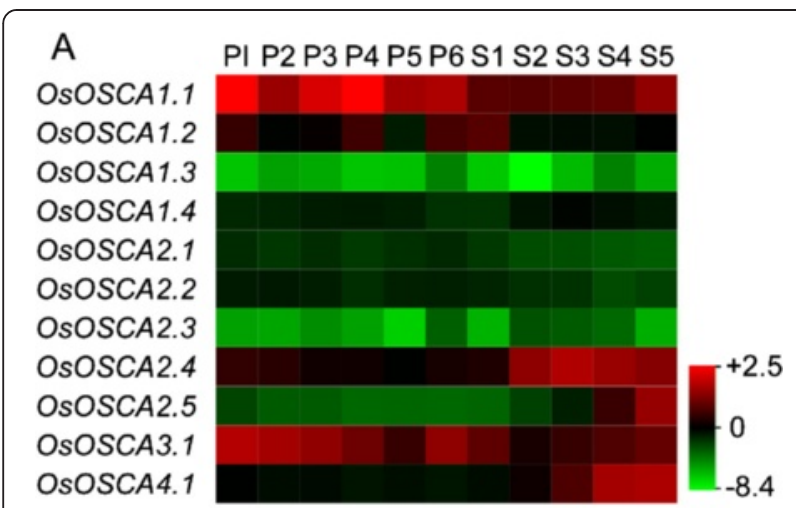

B
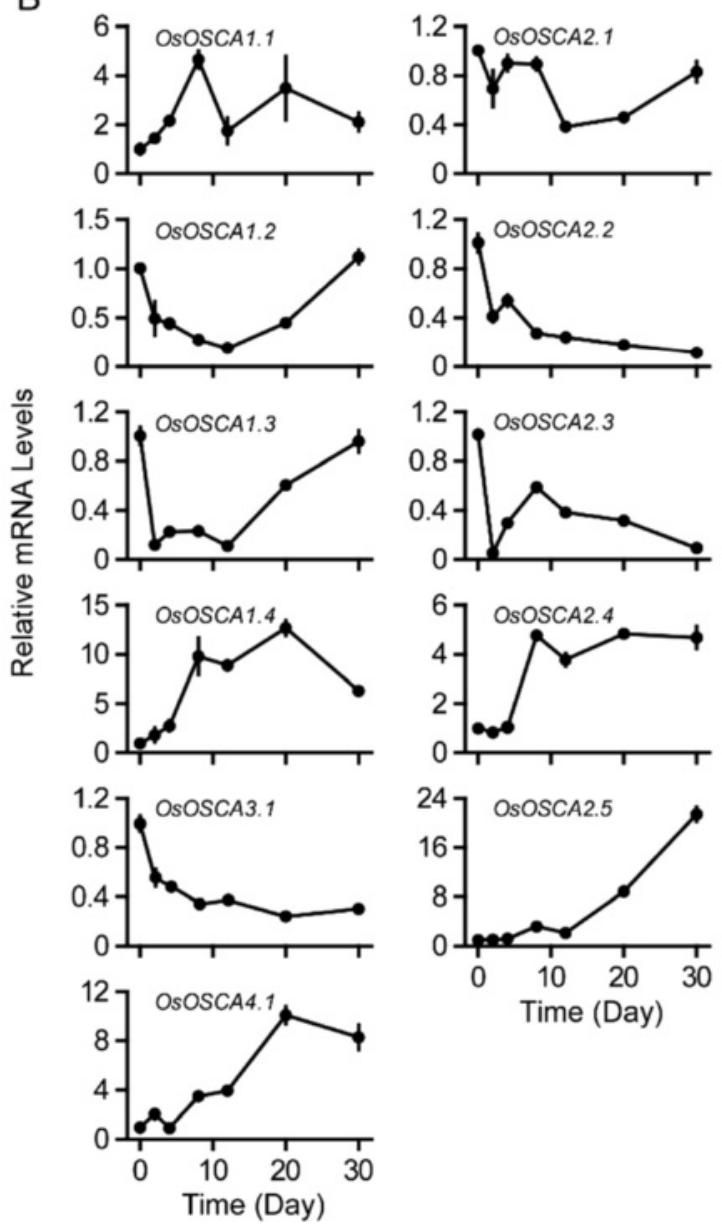

Fig. 4 Expression profiles of OsOSCA genes during panicle and caryopsis development. a. The microarray data sets (GSE6893) of OsOSCA gene expression in organs at various developmental stages were reanalysed (Additional file 3: Table S3). The average log signal values of OSOSCA genes are presented in the form of a heat map. The colour key represents average log2 expression values of OSOSCA genes. The samples are indicated at the top of each lane. The following stages of panicle development are indicated as follows: P1, 0-3 cm; P2, 3-5 cm; P3, 5-10 cm; P4, 10-15 cm; P5, 15-22 cm; and $P 6,22-30 \mathrm{~cm}$. The following stages of caryopsis development are indicated as follows: S1, 0-2 dap (day after pollination); S2, 3-4 dap; S3, 5-10 dap; S4, 11-20 dap; and S5, 21-29 dap. A colour scale representing the average log signal values is shown on the right. b. The expression levels of OSOSCA genes during caryopsis development were monitored using qRT-PCR. Samples were collected at $0,2,4,8,12,20$, and 30 dap. The relative water content in corresponding stages is shown in Additional file 6: Figure S2. The relative mRNA levels of individual genes were normalised to that of actin. Error bars indicate the standard deviations (SD) of three biological replicates

decreased during seed imbibition, which may lower the expression of OsOSCA1.1, OsOSCA1.2, OsOSCA2.1, OsOSCA2.4, OsOSCA2.5, and OsOSCA4.1. These results suggest that OSCAs play important roles during caryopsis development and seed imbibition.

In plants, circadian rhythms control stomatal conductance, transpiration, and relative water content around the guard cells, which regulates osmotic changes in the leaf [23]. Previously, we showed that $\mathrm{Ca}^{2+}$-sensing receptor (CAS) mediated the external $\mathrm{Ca}^{2+}\left(\left[\mathrm{Ca}^{2+}\right]_{\mathrm{o}}\right)$-induced $\left[\mathrm{Ca}^{2+}\right]_{\mathrm{i}}$ increase in guard cells and $\left[\mathrm{Ca}^{2+}\right]_{0}$-induced stomatal closure [33]. We further showed that $\left[\mathrm{Ca}^{2+}\right]_{i}$ oscillations were synchronised to $\left[\mathrm{Ca}^{2+}\right]_{\mathrm{o}}$ oscillations through the CAS/IP3 pathway in Arabidopsis thaliana [34]. In this study, we showed that the expression of OsOSCA1.2, OsOSCA2.1, and OsOSCA2.2 was orchestrated by the circadian clock, suggestive of their potential roles in sensing and responding to extracellular osmotic changes caused by circadian rhythms. Previous extensive research showed that plants respond and adapt to drought and high-salinity stresses by inducing the expression of a number of genes $[1,35]$. PEG, $\mathrm{NaCl}$, and drought stress are often interconnected and may induce similar cellular damage [36], as osmotic stress is the first and primary component of salt and drought stress upon exposure of plants to high $\mathrm{NaCl}$ concentrations and water-deficient environments [1]. And ABA, a key plant stress-signalling hormone, is synthesised in response to various abiotic stresses and regulates the expression of numerous stress-responsive genes in plants [37]. In this study, we found that the expression of eight OsOSCA genes was upregulated by at least one type of osmoticrelated abiotic stress, such as PEG, $\mathrm{NaCl}$, drought, or ABA treatment; in contrast, the expression of OsOSCA1.3 was 


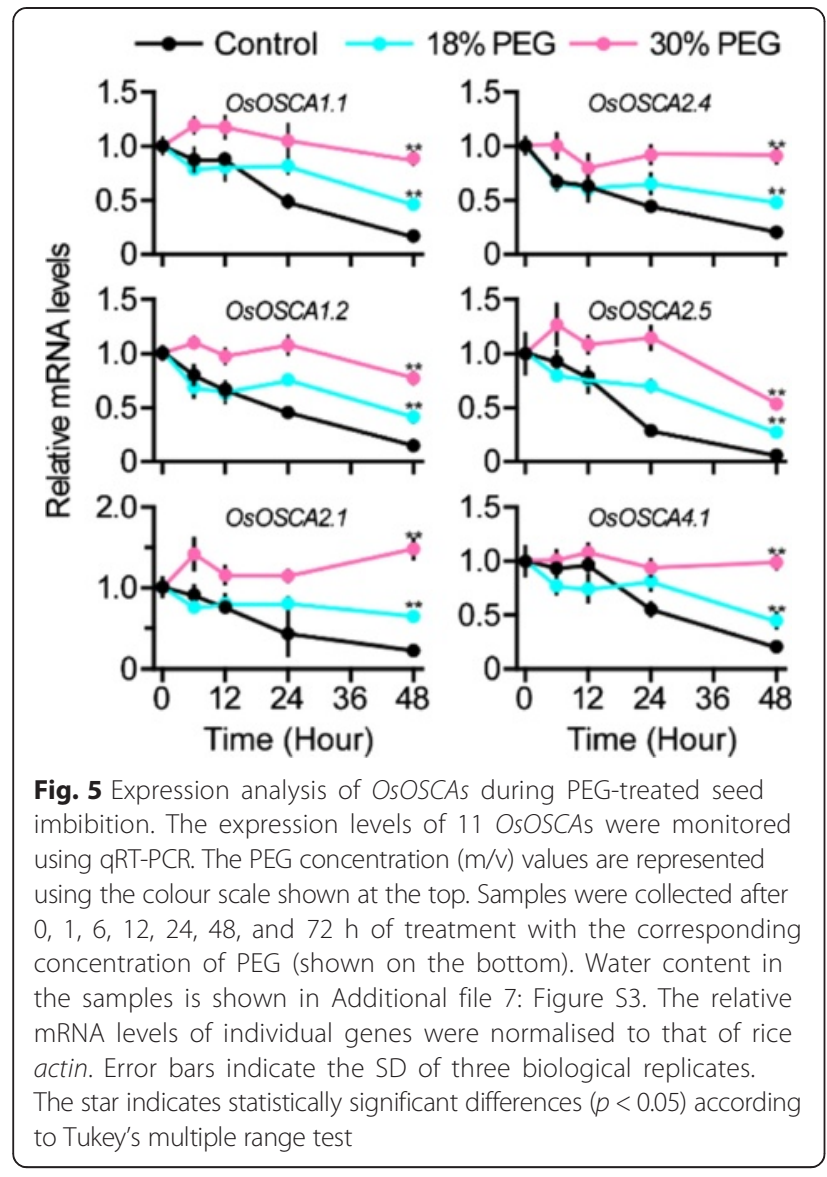

decreased by PEG and ABA treatment. We also found that OsOSCA2.2 and OsOSCA2.3 were not regulated by these four kinds of abiotic-related stress. In particular, the expression of OsOSCA2.2 was only showed as circadian rhythm oscillation, but not in osmotic-related abiotic stress, which indicated that OsOSCA2.2 plays a different role in sensing and responsing to water potential in guard cells. These results suggested that each member of the OsOSCA family plays a distinct role in the growth and development and the responses to diverse abiotic stresses, and provided further clues for the study of the physiological function of OsOSCAs as an osomosensor in rice.

\section{Conclusions}

OSCA was first characterised as an osmosensor that mediated hyperosmolality-induced $\left[\mathrm{Ca}^{2+}\right]_{\mathrm{i}}$ increases in Arabidopsis, indicating that this multiple-member family may play pivotal roles in sensing the exogenous and endogenous osmotic changes and in regulating plant growth and development. Sequence and phylogenetic analyses showed that 11 OsOSCAs from Oryza sativa L. Japonica contained a conserved DUF221 domain and shared common structural characteristics with their


Fig. 6 Circadian rhythmic expression of several OsOSCAs. Expression levels of OsOSCA1.2, OsOSCA2.1, and OsOSCA2.2 in shoots of four-leaf stage $\mathrm{ZH} 11$ seedlings under 14 -h light $\left(24^{\circ} \mathrm{C}\right) / 10$-h dark $\left(20^{\circ} \mathrm{C}\right)$ photoperiod conditions were analysed using quantitative real-time RT-PCR. Samples were collected every $3 \mathrm{~h}$. Data were normalised against actin expression. The expression of marker gene is shown in Additional file 8: Figure S4. Error bars indicate the SD of three biological replicates

homologs in Oryza sativa L. ssp. Indica, Oryza glaberrima, and Oryza brachyantha. In addition, we demonstrated that the expression of OsOSCAs was correlated with various exogenous and endogenous osmotic changes in an organ/tissue-specific manner in rice. 


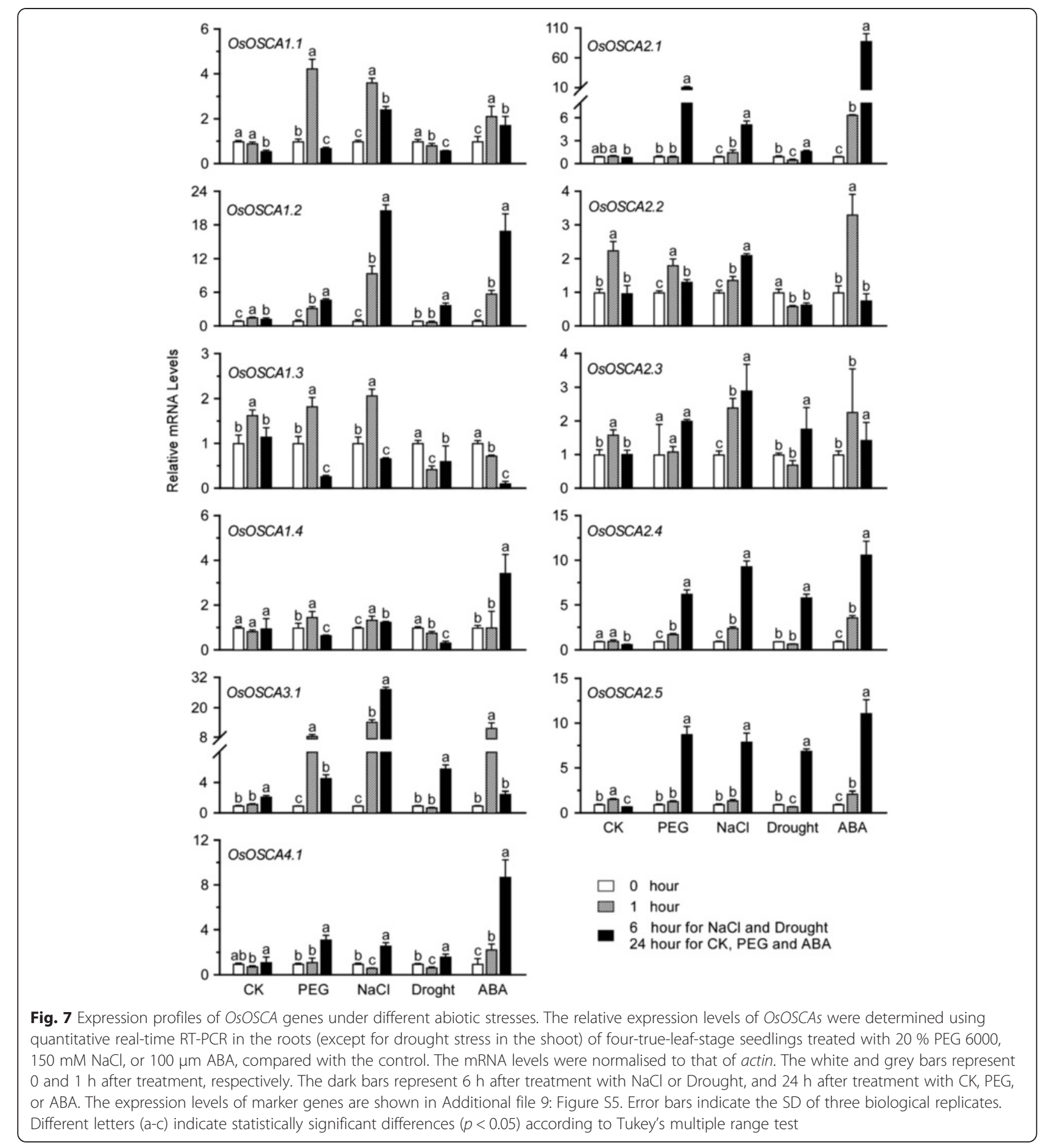

\section{Methods}

Plant material, growth conditions, and osmotic-related stress treatment

Rice plants (Oryza sativa L. spp. japonica cv. Zhonghua11) were planted in a growth chamber and in fields (from May to October, annually) at Beijing Normal University (Beijing, China). For growth in the chamber, seeds were incubated for at least 1 week at $42{ }^{\circ} \mathrm{C}$ to break any dormancy, and then soaked in water at $20^{\circ} \mathrm{C}$ for 3 days and germinated for 1 day at $37{ }^{\circ} \mathrm{C}$. The most uniformly germinated seeds were transferred into a 96-well plate, from which the bottom was removed. The plate was floated on water for 1 day at $37^{\circ} \mathrm{C}$ in the dark to promote root growth and then transferred into a growth chamber with a 14 -h light $\left(24^{\circ} \mathrm{C}\right) / 10$-h dark 
$\left(20^{\circ} \mathrm{C}\right)$ photoperiod. Five days later, the seedlings were cultured with Yoshida's culture medium, which was replaced every 2 days. For osmotic-related stress treatment, 30-dayold seedlings were separately transferred into Yoshida's culture medium containing $150 \mathrm{mM} \mathrm{NaCl}, 20 \%$ (w/v) PEG 6000, and $100 \mu \mathrm{M}$ ABA, with ethyl alcohol $(100 \mu \mathrm{L} /$ L final concentration) as a control. In addition, 30-day-old seedlings were placed in the growth chamber at 50-60\% relative humidity for drought-stress treatment.

For growth in the fields, the rice seeds were soaked in $1 \%$ carbendazim for 1 day and in water at $20{ }^{\circ} \mathrm{C}$ for $3-5$ days. Then, the most uniformly germinated seeds were sown in the seed bed until approximately the four-trueleaf stage. Seedlings were transplanted into the field and grown for about five months. Field management, including irrigation, fertiliser application, and pest control essentially followed normal agricultural practices. To analyse the expression pattern of OsOSCA genes, the following tissues and organs were collected: 30-day-old root $(\mathrm{Rt})$ and shoot $(\mathrm{St})$, mature stem $(\mathrm{Sm})$, mature flag leaf $(\mathrm{Fl})$, stamen $(\mathrm{Sn})$, pistil $(\mathrm{Pi})$, and mature seed (Sd, 45 days after pollination), the different stages of caryopsis development, including $0,2,4,8,12,20$, and 30 days after pollination, and seed imbibition at $0,1,6,12,24,48$, and $72 \mathrm{~h}$ at room temperature. All materials were collected at the indicated times, immediately frozen in liquid nitrogen, and stored at $-80{ }^{\circ} \mathrm{C}$ prior to RNA extraction.

\section{Identification of OSCAs in rice}

We used fifteen Arabidopsis OSCAs to perform a TBLASTN search of the rice genome database (http:// rice.plantbiology.msu.edu/) and obtained the cDNA and protein sequence of OsOSCA in Oryza sativa L. ssp. Japonica. OsOSCA orthologues of Oryza sativa L. ssp. Indica (OsIOSCAs), Oryza glaberrima (OgOSCAs), and Oryza brachyantha (ObOSCAs) were obtained from Ensembl Genomes (http://www.ensembl.org/index.html) using the best reciprocal BLAST software. The Simple Modular Architecture Research Tool (SMART; http://smart.emblheidelberg.de/smart/set_mode.cgi?NORMAL $=1$ ) was used to identify OSCAs with the presence of DUF221 and other typical domains in their protein structure. The general information and sequence characteristics of 45 OSCAs from four rice species are listed in Additional file 1: Table S1. Gene structures of OSCAs were analysed on the Gene Structure Display Server 2.0 (GSDS; http://gsds.cbi.pku.edu.cn/).

\section{Phylogenetic analysis and sequence alignment}

Full-length CDS sequences of OSCA genes from different rice species were aligned using the ClustalX 1.83 software [38] and the phylogenetic tree was constructed using MEGA v5 with the neighbour- joining method [39]. A total of 1,000 bootstrap replicates were performed in each analysis to obtain confidence support. The conserved protein domain of DUF221 in OsOSCAs was analysed using DNAMAN software (http://www.lynnon.com/dnaman.html) and modified manually [40]. The TM domains in OsOSCAs were annotated according to TMHMM Server v. 2.0 predictions (http://www.cbs.dtu.dk/services/ TMHMM/).

\section{RNA extraction and quantitative real-time PCR}

Total RNA was isolated from rice tissues using the TRI$\mathrm{zol}^{\circ}$ reagent (Invitrogen, USA) and purified using a PureLink $^{\circ}$ RNA Mini Kit (Invitrogen) combined with the PureLink ${ }^{\ominus}$ DNase kit (Invitrogen), according to the manufacturer's protocol. Approximately $4 \mu \mathrm{g}$ of total RNA was reverse-transcribed using the Reverse-Aid ${ }^{\text {tw }}$ First Strand cDNA Synthesis Kit to generate cDNA (Fermentas, Canada). Quantitative real-time PCR (qRT-PCR) was performed on the 7500 Fast Real-Time PCR System (Applied Biosystems, USA) using Power SYBR ${ }^{\circ}$ Green PCR Master Mix (Applied Biosystems). The thermal program was $2 \mathrm{~min}$ at $50{ }^{\circ} \mathrm{C}, 10 \mathrm{~min}$ at $95^{\circ} \mathrm{C}$, followed by $40 \mathrm{cy}$ cles of $15 \mathrm{~s}$ at $95{ }^{\circ} \mathrm{C}$ and $60 \mathrm{~s}$ at $60{ }^{\circ} \mathrm{C}$. The data were normalised to the rice actin gene (LOC_Os03g61970.1) [41] using the $\triangle \Delta C T$ method, as described previously [42]. To investigate whether the expression of actin is stability under different abiotic stresses, the expression of other two housekeeping gene eEF1a (LOC_Os03 g08020) and UBQ5 (LOC_Os01g22490) was detected compared to actin. Gene expression stability values (M) of these three genes were calculated using geNorm as described by Vandesompele et al. [43]. The dissociation curve program was used to confirm the specificity of the target amplification product. All primers used in this study are listed in Additional file 2: Table S2. At least three independent biological replicates were performed for qRT-PCR analysis. Value changes of more than twofold ( $>2$ or $<0.5$ ) were considered to indicate the induction or repression of OsOSCA expression. Analysis was performed using the Data Processing System, and a oneway analysis of variance (ANOVA) and Tukey's multiple range test [44] were conducted to determine significant differences. $P<0.05$ was considered to indicate statistical significance.

\section{In silico expression analysis of OsOSCAs}

OsOSCA gene microarray data were extracted from the Rice Functional Genomic Expression Database (http:// signal.salk.edu/cgi-bin/RiceGE) to analyse the expression profiles of OsOSCAs in organs during different developmental stages (GSE6893) (Additional file 3: Table S3). The absolute signal values were respectively divided by the average of all absolute values. 


\section{Availability of supporting data}

All relevant supporting data can be found within the supplementary files accompanying this article. Phylogenetic data supporting the results of this article are available in the TreeBASE repository, http://purl.org/phylo/ treebase/phylows/study/TB2:S17910.

\section{Additional files}

Additional file 1: Table S1. General information and sequence characterisation of 45 OSCA genes from Oryza sativa L. ssp. Japonica, Oryza sativa L. ssp. Indica, Oryza glaberrima, and Oryza brachyantha. (DOC $89 \mathrm{~kb}$ )

Additional file 2: Table S2. Sequences of oligonucleotide primers for qRT-PCR. F: forward; R: reverse. (DOC $52 \mathrm{~kb}$ )

Additional file 3: Table S3. Microarray data of OsOSCA expression patterns during panicle growth and caryopsis development in rice. (DOC $40 \mathrm{~kb}$ )

Additional file 4: Table S4. The relative expression of three housekeeping genes: actin, eEF1a and UBQ5 at different abiotic-related stress treatment condition was detected by qRT-PCR. (DOC $25 \mathrm{~kb}$ )

Additional file 5: Figure S1. Predicted conserved domains in OSCAs of Oryza sativa L. ssp. Japonica, Oryza sativa L. ssp. Indica, Oryza glaberrima, and Oryza brachyantha. (DOC $233 \mathrm{~kb}$ )

Additional file 6: Figure S2. Relative water content of rice caryopsis at different stages after pollination. (DOC $41 \mathrm{~kb}$ )

Additional file 7: Figure S3. The relative water content of ZH11 seeds during imbibition in solutions containing various PEG concentrations. (DOC $53 \mathrm{~kb}$ )

Additional file 8: Figure S4. Circadian rhythmic expression of the marker gene OsLHY in four-leaf-stage ZH11 seedlings. (DOC $56 \mathrm{~kb}$ )

Additional file 9: Figure S5. Expression of marker genes in roots of four-leaf-stage rice in the presence of osmotic-related abiotic stresses. (DOC $67 \mathrm{~kb})$

\section{Abbreviations}

DAF: days after flowering; HAl: hours after imbibition; OICl: hyperosmolalityinduced $\left[\mathrm{Ca}^{2+}\right]_{\mathrm{i}}$ increases; OSCA: hyperosmolality-gated calcium-permeable channels.

\section{Competing interests}

The authors declare that they have no competing interests.

\section{Authors' contributions}

YL, FY, ZW and SH conceived and designed the study. YL, FY, ZW, YL and FW conducted the experiments and analyzed the data. WZ, TZ and XJ helped to conduct experiment and data analysis. YW, $\mathrm{HZ}$ and ZMP revised the manuscript. $\mathrm{YL}$ and $\mathrm{SH}$ wrote the manuscript. All authors have read and approved the final version of the paper.

\section{Acknowledgements}

This work was co-supported by the Beijing Municipal Science and Technology Project Z121105002812042, the National Key Basic Research Program of China (973 Program) 2013CB126902, and the National Natural Science Foundation of China Grant 31070250.

\section{Author details}

'Beijing Key Laboratory of Gene Resource and Molecular Development, College of Life Sciences, Beijing Normal University, Beijing 100875, China. ${ }^{2}$ Department of Biology, Duke University, Durham, NC 27708, USA.

Received: 17 April 2015 Accepted: 22 October 2015

Published online: 26 October 2015

\section{References}

1. Shavrukov $Y$. Salt stress or salt shock: which genes are we studying? J Exp Bot. 2013;64(1):119-27

2. Ingram J, Bartels D. The molecular basis of dehydration tolerance in plants Annu Rev Plant Physiol Plant Mol Biol. 1996:47:377-403.

3. Zhu J-K. Salt and drought stress signal transduction in plants. Annu Rev Plant Biol. 2002;53:247-73.

4. Bartels D, Sunkar R. Drought and salt tolerance in plants. Crit Rev Plant Sci. 2005;24(1):23-58

5. Shinozaki K, Yamaguchi-Shinozaki K. Molecular responses to dehydration and low temperature: differences and cross-talk between two stress signaling pathways. Curr Opin Plant Biol. 2000;3(3):217-23.

6. Yamaguchi-Shinozaki K, Shinozaki K. Transcriptional regulatory networks in cellular responses and tolerance to dehydration and cold stresses. Annu Rev Plant Biol. 2006:57:781-803.

7. Yoshida T, Mogami J, Yamaguchi-Shinozaki K. ABA-dependent and ABA-independent signaling in response to osmotic stress in plants. Curr Opin Plant Biol. 2014;21:133-9.

8. Fujita Y, Fujita M, Shinozaki K, Yamaguchi-Shinozaki K. ABA-mediated transcriptional regulation in response to osmotic stress in plants. J Plant Res. 2011:124(4):509-25.

9. Kiyosue T, Yamaguchi-Shinozaki K, Shinozaki K. Cloning of cDNAs for genes that are early-responsive to dehydration stress (ERDs) in Arabidopsis thaliana L.: identification of three ERDs as HSP cognate genes. Plant Mol Biol. 1994:25(5):791-8

10. Rai A, Suprasanna P, D'Souza SF, Kumar V. Membrane topology and predicted RNA-binding function of the 'Early Responsive to Dehydration (ERD4)' plant protein. PloS ONE. 2012, 7(e326583). doi: 10.1371/ journal.pone.0032658. Epub 2012 Mar 14

11. Liu YH, Li HY, Shi YS, Song YC, Wang TY, Li Y. A maize Eearly responsive to dehydration gene, ZmERD4, provides enhanced drought and salt tolerance in Arabidopsis. Plant Mol Biol Report. 2009:27(4):542-8.

12. Camargo SR, Cancado GMA, Ulian EC, Menossi M. Identification of genes responsive to the application of ethanol on sugarcane leaves. Plant Cell Rep. 2007;26(12):2119-28

13. Knight $H$, Trewavas AJ, Knight MR. Calcium signalling in Arabidopsis thaliana responding to drought and salinity. Plant J. 1997;12(5):1067-78.

14. McAinsh MR, Pittman JK. Shaping the calcium signature. New Phytol. 2009;181(2):275-94

15. Booth $\mathbb{R}$, Edwards MD, Black S, Schumann U, Miller S. Mechanosensitive channels in bacteria: signs of closure? Nat Rev Micro. 2007;5(6):431-40.

16. Árnadóttir J, Chalfie M. Eukaryotic mechanosensitive channels. Annu Rev Biophys. 2010;39(1):111-37.

17. Yuan F, Yang $H$, Xue $Y$, Kong D, Ye R, Li C, et al. OSCA1 mediates osmotic-stress-evoked $\mathrm{Ca} 2+$ increases vital for osmosensing in Arabidopsis. Nature. 2014;514(7522):367-71.

18. Xu GX, Guo CC, Shan HY, Kong HZ. Divergence of duplicate genes in exon-intron structure. Proc Natl Acad Sci U S A. 2012;109(4):1187-92.

19. Schroeder BC, Cheng T, Jan YN, Jan LY. Expression cloning of TMEM16A as a calcium-activated chloride channel subunit. Cell. 2008:134(6):1019-29.

20. Chatzigeorgiou M, Bang S, Hwang SW, Schafer WR. tmc-1 encodes a sodium-sensitive channel required for salt chemosensation in C. elegans. Nature. 2013;494(7435):95-9.

21. Pan B, Geleoc GS, Asai Y, Horwitz GC, Kurima K, Ishikawa K, et al. TMC1 and TMC2 Are Components of the Mechanotransduction Channel in Hair Cells of the Mammalian Inner Ear. Neuron. 2013;79(3):504-15.

22. Dawson TE. Hydraulic lift and water use by plants: implications for water-balance, performance and plant-plant interactions. Oecologia. 1993:95(4):565-74.

23. Webb AA. The physiology of circadian rhythms in plants. New Phytol. 2003;160(2):281-303

24. Izawa T, Oikawa T, Sugiyama N, Tanisaka T, Yano M, Shimamoto K. Phytochrome mediates the external light signal to repress FT orthologs in photoperiodic flowering of rice. Genes Dev. 2002;16(15):2006-20.

25. Huang XY, Chao DY, Gao JP, Zhu MZ, Shi M, Lin HX. A previously unknown zinc finger protein, DST, regulates drought and salt tolerance in rice via stomatal aperture control. Genes Dev. 2009;23(15):1805-17.

26. Du H, Wang NL, Cui F, Li XH, Xiao JH, Xiong LZ. Characterization of the beta-Carotene Hydroxylase Gene DSM2 Conferring Drought and Oxidative Stress Resistance by Increasing Xanthophylls and Abscisic Acid Synthesis in Rice. Plant Physiol. 2010;154(3):1304-18. 
27. Igarashi Y, Yoshiba Y, Sanada Y, YamaguchiShinozaki K, Wada K, Shinozaki K. Characterization of the gene for Delta(1)-pyrroline-5-carboxylate synthetase and correlation between the expression of the gene and salt tolerance in Oryza sativa L. Plant Mol Biol. 1997;33(5):857-65.

28. Yang X, Yang YN, Xue LJ, Zou MJ, Liu JY, Chen F, et al. Rice ABI5-Like1 Regulates Abscisic Acid and Auxin Responses by Affecting the Expression of ABRE-Containing Genes. Plant Physiol. 2011;156(3):1397-409.

29. Hou C, Tian W, Kleist T, He K, Garcia V, Bai F, et al. DUF221 proteins are a family of osmosensitive calcium-permeable cation channels conserved across eukaryotes. Cell Res. 2014.

30. Ammiraju JS, Lu F, Sanyal A, Yu Y, Song $X$, Jiang N, et al. Dynamic evolution of Oryza genomes is revealed by comparative genomic analysis of a genus-wide vertical data set. Plant Cell. 2008;20(12):3191-209.

31. Vaughan DA, Morishima H, Kadowaki K. Diversity in the Oryza genus. Curr Opin Plant Biol. 2003;6(2):139-46.

32. Bradford KJ. Water-stress and the water relations of seed development - a critical review. Crop Sci. 1994;34(1):1-11.

33. Han SC, Tang RH, Anderson LK, Woerner TE, Pei ZM. A cell surface receptor mediates extracellular Ca2+ sensing in guard cells. Nature. 2003;425(6954):196-200.

34. Tang R-H, Han S, Zheng H, Cook CW, Choi CS, Woerner TE, et al. Coupling Diurnal Cytosolic Ca2+ Oscillations to the CAS-IP3 Pathway in Arabidopsis. Science. 2007;315(5817):1423-6.

35. Fujita Y, Yoshida T, Yamaguchi-Shinozaki K. Pivotal role of the AREB/ABF-SnRK2 pathway in ABRE-mediated transcription in response to osmotic stress in plants. Physiol Plant. 2013;147(1):15-27.

36. Wang W, Vinocur B, Altman A. Plant responses to drought, salinity and extreme temperatures: towards genetic engineering for stress tolerance. Planta. 2003;218(1):1-14.

37. Finkelstein R. Abscisic Acid synthesis and response. Arabidopsis Book. 2013;11.

38. Chenna R, Sugawara H, Koike T, Lopez R, Gibson TJ, Higgins DG, et al. Multiple sequence alignment with the Clustal series of programs. Nucleic Acids Res. 2003:31(13):3497-500.

39. Tamura K, Peterson D, Peterson N, Stecher G, Nei M, Kumar S. MEGA5: Molecular evolutionary genetics analysis using maximum likelihood, evolutionary distance, and maximum parsimony methods. Mol Biol Evol. 2011;28(10):2731-9.

40. $\mathrm{Ma} \mathrm{HL}$, Zhao J. Genome-wide identification, classification, and expression analysis of the arabinogalactan protein gene family in rice (Oryza sativa L.). J Exp Bot. 2010;61(10):2647-68.

41. Jain M. Genome-wide identification of novel internal control genes for normalization of gene expression during various stages of development in rice. Plant Sci. 2009;176(5):702-6.

42. Livak KJ, Schmittgen TD. Analysis of relative gene expression data using real-time quantitative $P C R$ and the $2-\Delta \Delta C T$ method. Methods. 2001;25(4):402-8.

43. Vandesompele J, De Preter K, Pattyn F, Poppe B, Van Roy N, De Paepe A, Speleman F. Accurate normalization of real-time quantitative RT-PCR data by geometric averaging of multiple internal control genes. Genome Biology. 2002;3(7):research0034.0031-research0034.0011.

44. Tang Q-Y, Zhang C-X. Data Processing System (DPS) software with experimental design, statistical analysis and data mining developed for use in entomological research. Insect Science. 2013;20(2):254-60.

\section{Submit your next manuscript to BioMed Central and take full advantage of:}

- Convenient online submission

- Thorough peer review

- No space constraints or color figure charges

- Immediate publication on acceptance

- Inclusion in PubMed, CAS, Scopus and Google Scholar

- Research which is freely available for redistribution 